\title{
Engineering Approximate Analysis Method of Rod Projectile Penetrating Concrete Target
}

\author{
Wang Feng ${ }^{1 *, 2}$, Jiang Yaqiong ${ }^{1}$, Ma Yurong $^{1}$, Li Dan $^{1}$ and Zhou Wei ${ }^{1}$ \\ ${ }^{1}$ School of Urban Construction, Anhui Xinhua University, Hefei 230088, China \\ ${ }^{2}$ Key Laboratory of Building Structure of Anhui Higher Education Institutes, Anhui Xinhua University, Hefei 230088, China
}

\begin{abstract}
The penetration process of rod projectile into concrete target is very complex, and the theoretical analysis is very difficult. In this paper, by using the engineering approximation method, through some reasonable simplification of the rod projectile penetration model, the analytical formulas of the penetration resistance, penetration depth and other important physical quantities are established. Through the calculation of penetration depth, when the sliding friction coefficient is 0.1 , the results are in good agreement with the experimental results. The results show that the higher the projectile density and the sharper the shape of the warhead, the stronger the penetration ability.
\end{abstract}

\section{Introduction}

Penetration is widely used in both military and civil fields. It is always the desire of weapon developers to analyze the penetration mechanism and effect of projecttile on concrete in theory. However, concrete is a kind of heterogeneous and anisotropic porous brittle material, its structure is more complex, and it shows the characteristics related to the strain rate under dynamic loading. The penetration of projectile into concrete target plate includes the dynamic process of large deformation, damage and failure. The damage and failure of material is very complex, which makes the theoretical analysis very difficult. In the current research methods, field experimental research is essential, but it costs a lot of money and time. Numerical simulation method has the advantages of high accuracy, and can use some complex constitutive models, but the method requires high programming or operation skills for users, so it is difficult for beginners to master. However, some common empirical formulas are difficult to be widely used in engineering practice due to their limited application scope. Relatively speaking, the engineering approximate analysis method has clear physical meaning, simple formula, clear results and certain accuracy, which is very suitable for the actual site use in engineering.

Jiao W.J studied the high-speed penetration of long rod, including the material properties of projectile target, the shape of long rod warhead, the effect of length diameter ratio and the design of segmented rod [1]. Yin Z.Y analyzes the approximate solution of AlekseevskiiTate model for ideal long rod penetration [2]. Deng J.J takes the penetration process of burst type tandem warhead's rear stage follow-up projectile into the pre perforated target as the research object. Based on the conical pre perforated and Coulomb friction model, the theoretical model of oval shaped projectile penetrating into the pre perforated target is developed and improved, including hole expanding or cratering and stable penetration [3]. Fan Z.J used shock wave theory to analyze the mechanism of transverse effect enhanced projectile penetrating metal target, and gave the approximate calculation formula of residual velocity after Pele colliding metal thin target [4]. Based on the force analysis of vertical penetration process and Newton's second law, Cheng X.L established the rigid body motion model of warhead, and obtained the variation law of various physical quantities in the process of vertical penetration by using numerical integration method [5].

In this paper, the engineering approximation method is used to simplify the model of the penetration problem of rod projectile, and the analytical formula of important physical quantities such as penetration resistance and penetration depth is established, and the analysis and verification is carried out by an example.

\section{Normal penetration resistance analy- sis of rod projectile}

When the rod projectile penetrates into the concrete target or hard soil target at medium velocity, the material of the projectile is much larger than that of the target, and the deformation of projectile is very small and can be ignored. For the convenience of studying the problem, the projectile target coordinate system $X O Z$ is established with the initial impact point of the projectile on the target as the origin $O$, the vertical downward direction as the $Z$ axis and the horizontal direction as the $X$ axis, as shown in Fig. 1. Let the initial impact velocity of the projectile be $V_{0}$, the penetration velocity at any time be $V_{Z}$, and the projectile vertically invades the target. The resistance of rod projectile in the process of

\footnotetext{
* Corresponding author: wangfeng0706 @163.com
} 
penetration can be divided into two parts: the resistance of warhead and the resistance of rod sidewall. In order to simplify the calculation, we make the following assumptions.

(1) The projectile is a rigid body and does not deform during penetration.

(2) When the penetration velocity is low, the influence of side wall resistance on penetration depth can be ignored.

(3) The projectile is symmetrical about the $X O Z$ plane during penetration.

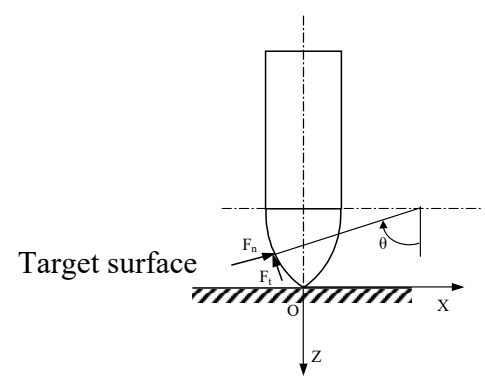

Fig. 1. Resistance of projectile.

Based on the cavity expansion theory, the resistance of rod projectile in the process of penetration can be obtained,

$$
F_{z}=\alpha_{i}+\beta_{i} V_{z}^{2}
$$

Where $\alpha_{i}$ and $\beta_{i}(i=1,2,3)$ are coefficients of different warhead shapes.

The shape coefficients are analyzed for the spherical warhead, the oval warhead and the cone warhead, as shown in Fig. 2.
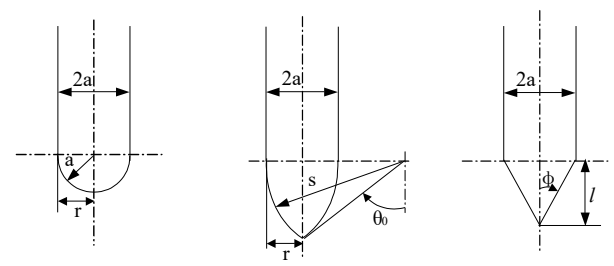

(a) spherical warhead

(b) oval warhead

(c) conical warhead

Fig. 2. Straight rod projectiles with three different shape warheads.

(1) Spherical warhead $(i=1)$

$$
\alpha_{1}=\pi a^{2} K A\left(1+\frac{\pi}{2} \mu_{m}\right), \quad \beta_{1}=\pi a^{2} B \rho\left(\frac{1}{2}+\frac{\pi}{8} \mu_{m}\right)
$$

Where $\rho$ is the density of the target medium, $K$ is the bulk modulus of the target material, A and B are the target material constant, $\mu_{m}$ is the coefficient of sliding friction, and $a$ is the radius of elastic rod.

(2) Oval warhead $(i=2)$

$$
\left\{\begin{aligned}
\alpha_{2}= & \pi K A\left[a^{2}+\mu_{m} s^{2}\left(\frac{\pi}{2}-\theta_{0}\right)-\mu_{m}(s-a) \sqrt{a(2 s-a)}\right] \\
\beta_{2}= & \pi B \rho\left[\frac{a^{3}(4 s-a)}{6 s^{2}}+\mu_{m} \frac{s^{2}}{4}\left(\frac{\pi}{2}-\theta_{0}\right)\right. \\
& \left.\quad-\mu_{m} \frac{(s-a)\left(3 s^{2}+4 s a-2 a^{2}\right) \sqrt{a(2 s-a)}}{12 s^{2}}\right] \\
\theta_{0}= & \sin ^{-1}\left(\frac{s-a}{s}\right)
\end{aligned}\right.
$$

Where $s$ is the radius of the arc of the oval warhead, $\theta_{0}$ is the central angle.

(3) Conical warhead $(i=3)$

$$
\left\{\begin{array}{l}
\alpha_{3}=\pi a^{2} K A\left(1+\mu_{m} \tan \phi\right) \\
\beta_{3}=\pi a^{2} B \rho \sin ^{2} \phi\left(1+\mu_{m} \tan \phi\right)
\end{array}\right.
$$

where $l$ is the length of the warhead and $2 \phi$ is the cone apex angle of the warhead, $\tan \phi=a / l$.

\section{Analysis of penetration depth of rod projectile}

According to Newton's second law, it can be known that the differential equation of motion when the rod projectile is penetrating forward is,

$$
M \frac{d V_{z}}{d t}=M V_{z} \frac{d V_{z}}{d z}=M g-F_{z}=M g-\left(\alpha_{i}+\beta_{i} V_{z}^{2}\right)
$$

where $M$ is the mass of the projectile and $M g$ is the weight of the projectile.

The size of $\mu_{m}$ in the actual penetration process should be related to the penetration velocity $V_{Z}$ and the performance of projectile target material. $\mu_{m}$ is usually a piecewise function related to the penetration velocity $V_{Z}$, which makes it difficult to calculate the penetration depth. In order to simplify the calculation process, we assume that $\mu_{m}$ is a constant in the penetration process. The initial condition and termination condition are,

$$
\left\{\begin{array}{lll}
t=0, & z=0, & V_{z}=V_{0} \\
t=t_{\max }, & z=z_{\max }, & V_{z}=0
\end{array}\right.
$$

By integrating equation (6), the final penetration depth of rod projectile is obtained,

$$
H=z_{\text {max }}=\frac{M}{2 \beta_{i}} \ln \left(1+\frac{\beta_{i} V_{0}^{2}}{\alpha_{i}-M g}\right)
$$

Now let's determine the material parameters in the formula. The target material constants $A$ and $B$ in formula (2)-(4) are given in reference [6], 


$$
\left\{\begin{array}{l}
A=\frac{2}{3} \frac{\tau_{0}}{K}\left[1-\ln \left(\eta^{*}\right)\right] \\
B=\frac{1}{\gamma^{2}}\left[\frac{3 \tau_{0}}{E}+\eta^{*}\left(1-\frac{3 \tau_{0}}{2 E}\right)^{2}+\frac{3\left(\eta^{*}\right)^{2 / 3}-\eta^{*}\left(4-\eta^{*}\right)}{2\left(1-\eta^{*}\right)}\right] \\
\gamma=\left[\left(1+\frac{\tau_{0}}{2 E}\right)^{3}-\left(1-\eta^{*}\right)\right]^{1 / 3}
\end{array}\right.
$$

where, $\eta^{*}$ is the lock strain volume strain, $E$ is the elastic modulus of the target material, and $\tau_{0}$ is the confined shear strength of the target material.

The above formula is substituted into the coefficient formula of warhead shape, and the coefficients $\alpha_{i}$ and $\beta_{i}$ $(i=1,2,3)$ can be determined. Therefore, the penetration depth of rod projectile with different shape in the target body can be determined by formula (7).

\section{Example analysis}

The experimental data of penetration depth of oval rod projectile into concrete are given in reference [7], as shown in Table 1. In the table, $d$ is the diameter of rod, $M$ is the mass of rod, $v$ is the impact velocity of projectile, and $H$ is the penetration depth.

Table 1. Experimental data of penetration depth.

\begin{tabular}{|c|c|c|c|c|}
\hline$d / \mathbf{m m}$ & Length diameter ratio & $M / g$ & $v /(\mathbf{m} / \mathbf{s})$ & $H / \mathbf{m m}$ \\
\hline \multirow{7}{*}{10} & \multirow{7}{*}{10} & 58 & 330 & 145 \\
\hline & & 56.5 & 372 & 123 \\
\hline & & 58 & 382 & 175 \\
\hline & & 58 & 420 & 205 \\
\hline & & 58 & 450 & 205 \\
\hline & & 58 & 494 & 277 \\
\hline & & 58 & 560 & 335 \\
\hline \multirow{5}{*}{40} & \multirow{5}{*}{15} & 6180 & 253 & 620 \\
\hline & & 6210 & 305 & 870 \\
\hline & & 6200 & 355 & 1120 \\
\hline & & 6200 & 403 & 1400 \\
\hline & & 6190 & 448 & 1690 \\
\hline \multirow{7}{*}{20} & \multirow{7}{*}{10} & 460 & 206 & 145 \\
\hline & & 461 & 252 & 210 \\
\hline & & 460 & 312 & 248 \\
\hline & & 462 & 367 & 327 \\
\hline & & 459 & 408 & 357 \\
\hline & & 460 & 434 & 437 \\
\hline & & 460 & 475 & 475 \\
\hline \multirow{4}{*}{20} & \multirow{4}{*}{10} & 459 & 500 & 536 \\
\hline & & 461 & 545 & 628 \\
\hline & & 460 & 602 & 730 \\
\hline & & 458 & 657 & 863 \\
\hline
\end{tabular}

By using equation (7), the final penetration depth of an oval rod projectile impacting a concrete target at an initial velocity of $0-800 \mathrm{~m} / \mathrm{s}$ is obtained. The comparison with the experimental data provided in reference [7] verifies the correctness of the theoretical formula proposed in this paper. The rod radius, warhead radius and projectile mass are all taken as test data $(\mathrm{CRH}=3.0)$. In this case, the projectile mass in formula (7) should be the sum of rod mass in Table 1 and calculated warhead mass. The concrete material parameters are as follows: bulk modulus $K=6.7 \mathrm{GPa}$, density $\rho=2240 \mathrm{~kg} / \mathrm{m}^{3}$, elastic modulus $E=11.3 \mathrm{GPa}$, shear strength of 4 months $\tau_{0}=95 \mathrm{MPa}$, lock strain compression volume strain $\eta^{*}=0.04$.

Fig. 3 shows the relationship between the penetration depth $H$ and the initial velocity $V_{0}$ when the projectile diameter $d$ and the sliding friction coefficient $\mu_{m}$ are different. The discrete data points in the figure correspond to the experimental results shown in Table 1, and the curve is the corresponding calculation results. It can be seen from the figure that the sliding friction coefficient $\mu_{m}$ has a great influence on the penetration depth. The increase of $\mu_{m}$ will lead to the increase of the tangential friction on the warhead surface and the increase of the axial resistance on the warhead, so the penetration depth decreases accordingly, which is consistent with the actual penetration process. When $\mu_{m}=0.1$, the penetration depth calculated in this paper is in good agreement with the experimental results, which is close to the sliding friction coefficient recommended in reference [6].

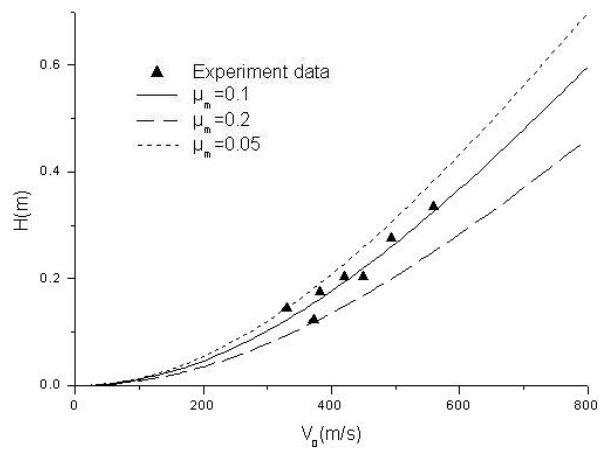

(a) $d=10 \mathrm{~mm}$

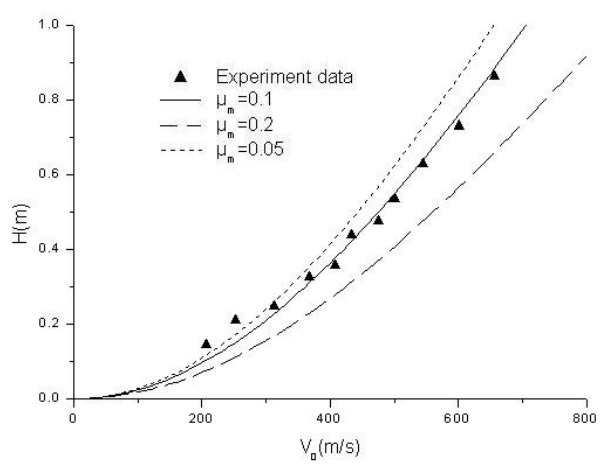

(b) $d=20 \mathrm{~mm}$

Fig. 3. Relationship between penetration depth and penetration velocity of projectile with different values of $\mu_{\mathrm{m}}$.

Fig. 4 shows the influence of different projectile mass $M$ on penetration depth $H$. The shape of the projectile is still oval warhead with $a=10 \mathrm{~mm}(\mathrm{CRH}=3.0)$. It can be seen from the figure that the projectile mass has a great influence on the penetration depth, so the 
warhead with higher density has stronger penetration ability.

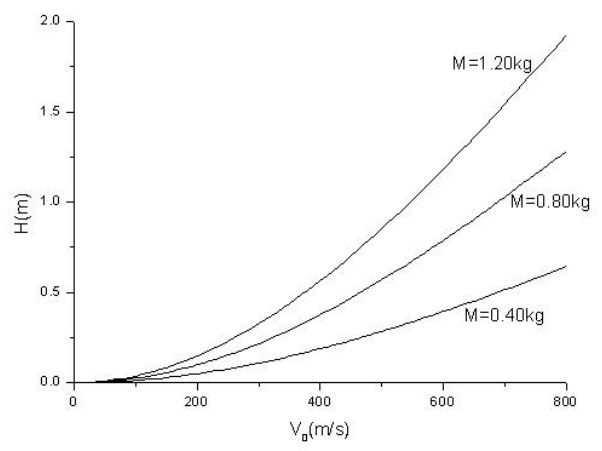

Fig. 4. Relationship between projectile mass $M$ and penetration depth $H$

Fig. 5 shows the relationship between the penetration depth $H$ and the initial velocity $V_{0}$ when the warhead shape is conical, oval and spherical respectively. The diameter of the warhead is $a=10 \mathrm{~mm}$, the CRH of the oval warhead is 3.0, and the height of the conical warhead is the same as that of the oval warhead. It can be seen from the figure that under the same conditions, the penetration ability of conical rod projectile is the strongest, while that of spherical rod projectile is the weakest. This is because at the same penetration velocity, the sharper the shape of the warhead, the smaller the penetration resistance and the greater the penetration depth. The smoother the shape of the warhead is, the greater the penetration resistance is and the smaller the penetration depth is.

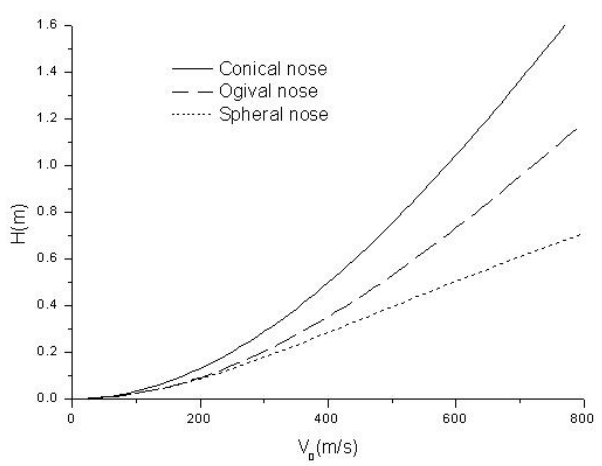

Fig. 5. Relationship between warhead shape and penetration depth $H$

In addition, the penetration depth of oval rod projectile penetrating concrete target is calculated by using the projectile model and material parameters described in reference [8], and the results are compared with those of numerical simulation, as shown in Fig. 6. In contrast, when $\mu_{m}=0.1$, the penetration depth calculated by approximate analysis is in good agreement with the numerical simulation results. There is a certain deviation between the approximate calculation and the simulation results in the low velocity and high velocity sections, which indicates that the simplified model used in the approximate analysis needs to be further improved.

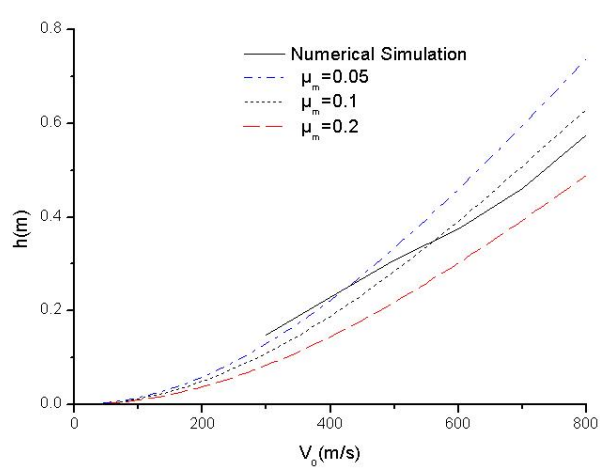

Fig. 6. Comparison between approximate analysis and numerical simulation of projectile penetration depth

\section{Conclusions}

In this paper, based on the cavity expansion theory, the analytical expressions of the axial resistance of three kinds of rod projectiles with different shapes are given by simplifying the model, and the analytical expressions of the penetration depth are obtained by Newton's second law. Through the calculation of penetration depth, when the sliding friction coefficient is 0.1 , the results are in good agreement with the experimental results. The results show that the higher the projectile density and the sharper the shape of the warhead, the stronger the penetration ability. The engineering approximate analysis method is simple in calculation, and it is an important method to study the penetration problem. However, there is still a certain gap between the engineering approximate analysis method and the actual penetration process. The following aspects need to be further studied。

(1) The engineering approximate analysis method is mainly used to calculate the penetration depth of long rod high-speed penetration, and the hole diameter, residual projectile length and mass need to be further studied

(2) The influence of material properties of projectile and target on high-speed penetration of long rod is deeply analyzed, and the relationship between target resistance and impact velocity is further studied.

(3) Continue to study the aspect ratio effect and its mechanism, and establish the theoretical analysis model which can reflect the aspect ratio effect.

In a word, the in-depth study of engineering approximate analysis method requires the development of experimental technology, the use of more advanced equipment and technology to verify the experiments of traditional projectile target combination, and the development of large velocity range experiments for new projectile target materials, different warhead shapes, impact attitude and target structure to verify the experimental phenomena and discover new penetration phenomena and deformation failure mechanisms. There is still a long way to go for the development of engineering approximate analysis method. 


\section{Acknowledgements}

This paper is one of the phased achievements of the key project of Natural Science Research in Universities of Anhui Province "Study on dynamic mechanical properties of heterogeneous materials based on extended finite element method" (KJ2019A0882), Anhui Province Quality Engineering Project "Teaching team of civil engineering" (2019jxtd118), Universities Quality Engineering Project "Curriculum system construction and teaching reform of structural mechanics" (2019jy031), and key project of Humanities and Social Sciences in Universities of Anhui Province "Research on digital restoration and protection technology of Huizhou color painting" (SK2020A0607).

\section{References}

1. Jiao W.J, Chen X.W. (2019) Research progress of long rod high speed penetration. Mechanics progress, 49: 312-391. (in Chinese)

2. Yin Z.Y, Chen X.W. (2021) Analysis of characteristic control parameters of long rod high speed penetration. Explosion and impact, 41: 140146. (in Chinese)

3. Deng J.J, Zhang X.F, Qiao Z.J, Guo L, He Y, Chen D.D. (2016) Theoretical analysis of penetration of pre perforated targets by oval projectiles. Explosion and impact, 36: 625-632. (in Chinese)

4. Fan Z.J, Ran X.W, Tang W.H, Yu G.D, Li Z.B, Chen W.K. (2015) Approximate calculation and analysis of axial residual velocity of Pele penetrating metal thin target. Journal of National Defense University of science and technology, 37 : 84-90. (in Chinese)

5. Cheng X.L, Zhao H, Li L.C, Ye H.F. (2019) The model of vertical penetration target action based on mechanical vibration theory. Explosion and impact, 39: 104-113. (in Chinese)

6. Xu J.B, Lin J.D, Tang R.D, Chu Z. (2002) Experimental study on long rod projectile penetrating concrete. Explosion and impact, 22: 174-178. (in Chinese)

7. M.J.Forrestal. (1986) Penetration into Dry Porous Rock. Int J Solids Structure, 22: 1485-1500.

8. Wang F. (2007) Study of finite element method and application for high velocity impact. China University of science and Technology Press, Hefei. (in Chinese) 